\title{
The Potential Use of an Autostereoscopic 3D Display in Microsurgery
}

\author{
P. Chios ${ }^{1}$, A. C. Tan ${ }^{1}$, A D Linney ${ }^{1}$, G. H. Alusi ${ }^{1}$, A. Wright ${ }^{1}$, \\ G. J. Woodgate ${ }^{2}$, and D. Ezra ${ }^{2}$ \\ ${ }^{1}$ Institute of Laryngology \& Otology \\ University College London \\ 330-332 Gray's Inn Road, London WC1X 8EE \\ doros@james.ilo.ucl.ac.uk \\ http://www.ilo.ucl.ac.uk \\ ${ }^{2}$ Sharp Laboratories Europe Ltd. \\ Edmund Halley Road, Oxford Science Park, Oxford OX4 4GB \\ http://www.sle.sharp.co.uk
}

\begin{abstract}
This paper investigates the potential use of a prototype micro-optic twin Liquid Crystal Display (LCD) monitor for stereomicroscopy in microsurgery. The new device displays stereoscopic images via a pair of colour Charge-Coupled Device (CCD) video cameras attached on to a surgical stereo microscope. The paper illustrates the 3D microscope-display system set-up and calibration for stereo viewing. A series of experimental techniques was devised to measure the user-response of the new display system in depth perception of a solid object against the traditional eyepieces of a stereo microscope. As a control, free viewing with the un-aided eyes was also measured. The positional data were collected using a passive mechanical arm. The results showed good correspondence between all three visualisation methods. Error analysis of our numerical findings suggests that the depth accuracy of the new device is well within the precision limits of hand movement for surgical operations. Our study also proves that there are small discrepancies within the sample population of operators using the system. A study based on the psychological and psychophysical factors influencing the system is planned.
\end{abstract}

\section{Introduction}

The research group from the Institute of Laryngology and Otology at UCL has been conducting active research in the field of Augmented Reality in Ear, Nose \& Throat (ENT) surgery. The aim of the project is to create a tool based on the concept of 'augmented reality' which will allow surgeons to realistically plan, rehearse and execute complex otological and base of the skull surgery. The scientific and technical issues relate to creating dynamic 3D images and involve real time processing, image buffer updating, parallel processing, developing, integrating an accurate tracking technique and creating a suitable human-machine interface for interacting under virtual surgical environment. This paper investigates the display aspect of the project, for which a binocular stereoscopic surgical microscope is transformed with "electronic eyes" to couple with a "3D TV" for surgery. 


\subsection{Stereo Vision}

Stereo Vision is important for the perception of depth. Each eye uses different images of the same object to form a solid view in the human visual system. The 3D view is achieved by using many of the depth cues such as scenes hidden by opaque objects lying closer to the observer, foreshortening of distant objects by perspectives, shadows cast by oblique illumination, shading of the surface luminance, rotation of the object, and binocular parallax. In stereo microscopy most of these cues disappear and the main one used for perceiving depth is the binocular parallax. This is vital in microsurgery where depth perception is very important.

\subsection{Microsurgery}

Microsurgery has always been considered as one of the most difficult and delicate types of surgery. This is mainly due to the limited amount of working space and the high density of anatomical structures that exist in the region of interest. The use of binocular operating stereo microscopes is universal, but they limit the surgeon's head movement. This often makes the surgical operation stressful, uncomfortable for the eyes and hence more hazardous for the patient.

The arrangement of devices, tables and stools inside the operating theatre is fairly standard and has not been changed for a long time. Operating ergonomics are essential for any type of operation, and together with health safety protocols, form the design requirements for introducing new devices to the operating theatre.

\subsection{Autostereoscopic 3D Displays}

The display of stereoscopic images has been the subject of research for many years. Early techniques involved the use of two genlocked, monochrome video cameras which, being attached onto a stereo microscope, could capture the anaglyph created by the microscope's objective lens and intermediate red or green filters. With the use of complementary colour glasses the observer could see an intermediate-magnified stereo image. Recently developed stereoscopic displays differ only a little from the original concepts. Such display systems suffer from uncomfortable eye-wear, reduced image brightness, image flicker and cross-talk levels up to $10 \%$ [1].

Autostereoscopic displays that do not require specialised headsets are considered as a more realistic approach to $3 \mathrm{D}$ viewing. They offer a greater viewing freedom than the binocular stereo microscope eyepieces and are also useful for multi-view image presentationt. However, image quality can be affected by several factors. Cross-talk of the two image channels may occur due to aberrations of the optical system. A limited display bandwidth causes degradation of image quality which results in lower depth perception. Additionally, it restricts the number of views that can be simultaneously displayed. The latter problem can be overcome by using observer tracking displays [2]. The observer's position is normally measured by infra-red or video image processing tracking devices. 
The current trend in autostereoscopic 3D display development is found in the use of thin film transistor Liquid Crystal Displays. These offer significant advantages such as flatness and thinness, high image resolution, high image contrast and fidelity, good colour quality and low cost. Especially 'Twin-LCD' systems are considered of great prospect as they can offer full resolution of the LCD to each to the observer's eye [3].

\section{3D Video Microscope}

The surgical stereo microscope, one of the main instruments used by the microsurgeons at the time of operation, is usually attached to the end of a side arm of a crane that stands at one side of the operating theatre. The microscope itself is small, light and compact so that it can move freely in all directions.

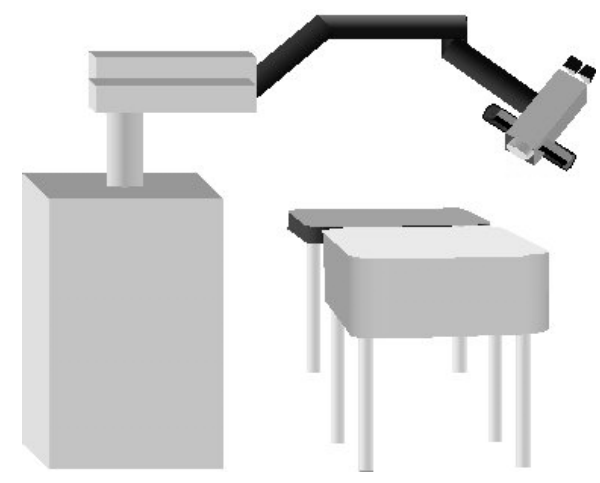

Figure 1: Typical microscope positioning inside the operating theatre

With new demands being introduced to medicine, the microscope structure has changed from its original form. For example, the need for teaching and subsequently for documentation brought forward the idea of splitting the light beam that comes out of the objective lens of the microscope into two identical beams. The first beam then follows its original optical path and enters the eyepiece while the other is directed towards a photographic camera, positioned at the focal plane of the light beam. This photographic camera mounting can be replaced with a video camera-TV adapter arrangement, through which the optical image observed by the microscope can be displayed on a 2D monitor or even recorded on a standard video recorder. In this way, staff inside and outside the operating theatre (e.g. consultants, medical students, nurses, etc.) can watch the procedure as if looking through one eye of the stereo microscope. 


\subsection{CCD Technology}

One of the latest advances in the field of video microscopy is based on the recent developments of Charge-Coupled Device (CCD) video cameras. Modern CCD cameras come in a range of sizes designated by the 'inch' notation. The most common ones are the 1/3,1/2,2/3 and 1 inch. The field of view of the microscope is determined by the projected image from the eyepiece and is generally larger than the CCD sensor size. For high resolution and colourful visualisation of the images we use the three chips, 1/2 CCD, C-mount Panasonic GP-US522 remote head video camera. The $1 / 2$ inch CCD covers only $20 \%$ of the total field of view, but offers a highly magnified video image. The 3 CCD sensors acquire the primary colours (Red, Green, Blue RGB) separately, thus leading to good colour representation.

\subsection{Camera Mounting}

The synthesis of stereoscopic images, captured from a binocular operating stereo microscope can be achieved by placing two video cameras at the distal ends of the beam splitter respectively. The monoscopic images relate to the left and right eye fields of view. When combined and calibrated correctly the two images form a stereoscopic image that can be displayed by an autostereoscopic 3D display monitor. A 10.4-inch prototype Sharp Micro-optic twin-LCD display monitor (courtesy of Sharp Laboratories Europe Ltd) was used to conduct our experimental study. The light beam leaving the microscope's beam splitter is originally focused at infinity. In order to focus the beam to a reasonable distance from the splitter's distal end we use a Zeiss f60 TV adapter. The adapter has manual fine focus, iris selection and a bayonet connection at the camera end.

The precise placement of the CCD active sensing area to focus onto the field of view of each of the microscopic output areas requires careful calibration. A small deviation of $1 \mathrm{~mm}$ from the ideal position can result to a distortion of $2 \mathrm{~cm}$ between identical monoscopic images even at low magnification factors (e.g. x10). Therefore, the correct alignment of the two cameras on the autostereoscopic 3D display requires prior calibration before the cameras are aligned vertically and horizontally using the 3D display itself.

To overcome the above problems we designed and manufactured a prototype coupler that can give a travelling distance for the horizontal $x-y$ plane of $\pm 2.5 \mathrm{~mm}$ and on the $z$ direction $\pm 2.5 \mathrm{~cm}$. The $x-y$ translation ensures fine adjustment of the two monoscopic images on the screen, while the $z$ direction movement finds accurately the ideal focusing position of the two cameras before or after the focal point. The coupler has a bayonet base so that it can be coupled to the TV adapter of the microscope. The other end is a C-mount coupling for video camera attachment. It can be locked at any desired position and can afford a maximum camera weight of up to 500 gr. The remote head video camera has negligible weight and does not affect the microscope's overall shape. 

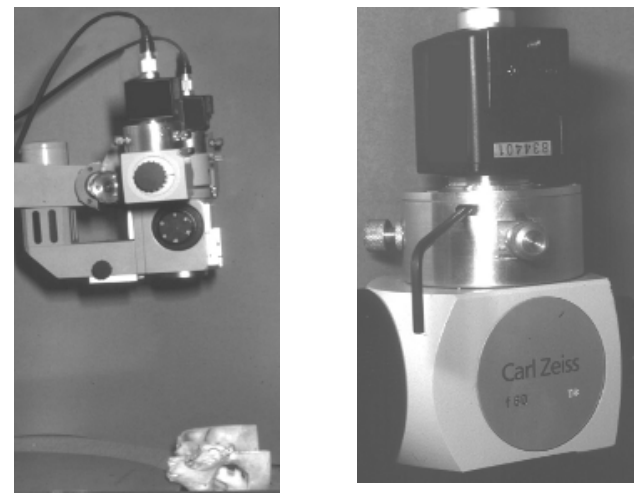

Figure 2: 3D Microscope profile and coupler close-up

\section{Prototype Experiment}

To test the effectiveness of the prototype Sharp Micro-Optic Twin-LCD monitor as an alternative to the eyepieces of the binocular operating stereo microscope, an observer-response evaluation test was devised. To conduct the experiment, we used a passive robot arm [4] to correctly identify and mark three identifiable points of a miniature model placed under the microscope. We chose 7 test subjects (including the author who is a medical physicist, the rest are all ENT surgeons) to carry out the experiment. The choice of examiners was made on criteria such as ease with microscope use and understanding of the visual information as it appears on the 3D display. A second series of experiments looked into the timed response of the observers on the task of passing a thread through the eye of a needle. In both experiments the microscopic image was viewed through both the 3D-display screen and the eyepieces. All experiments were conducted under a $x 16$ magnification factor.
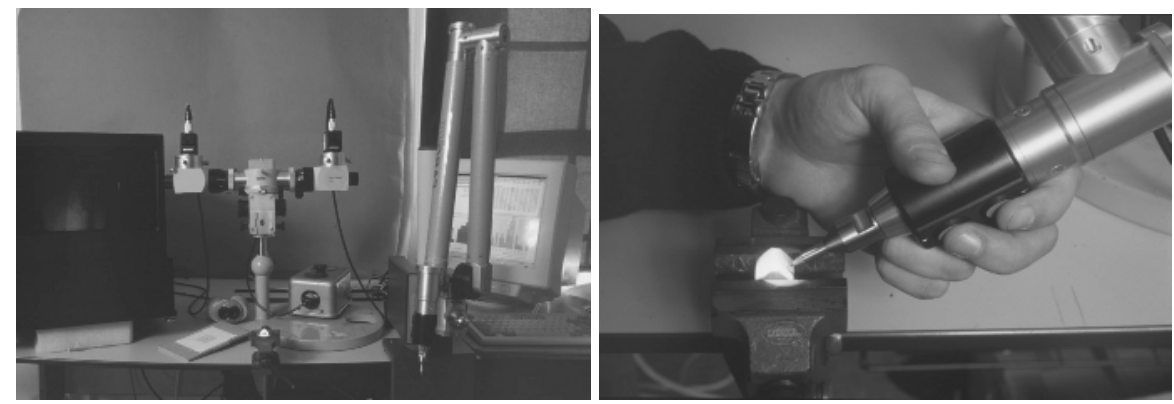

Figure 3: (a) Experimental arrangement of equipment (b) miniature model under the microscope 


\subsection{Use of 3D Mechanical Arm for Digitising Positions}

The passive robot arm used in our experiment is a multiple axis-articulated arm with a spherical working volume of 2.4 metres. It has a 0.25 inch ball probe attached to its working end and a 1-inch reference sphere placed at its base. The mechanical arm is counterbalanced and temperature compensated, with six or seven degrees of freedom. It has optical encoders, placed at each of six joints, which combine to provide complete point position $(x-y-z)$ and orientation $(i-j-k)$. Three-dimensional digitised measurements are taken between the distance of the ball probe and the origin at the reference sphere. It operates using a standard PC and can be used as a standalone 3D-measurement system. The 3D mechanical arm has an accuracy of \pm 0.075 $\mathrm{mm}$ and has a calibration procedure to maintain correct operation.

\subsection{Calibrating The System}

Calibration of the 3D microscope-display-arm system is carried out manually and in sequence of steps before the start of the experiment.

Initially, the focusing level of each camera is individually adjusted by placing an edge-enhanced object under the microscope. At each step, the cameras are alternatively selected to adjust to the same focus level. Therefore, both cameras have the same zooming and focusing levels.

The next calibration step involves the alignment of the two monoscopic images on the autostereoscopic 3D display. This is done with the use of a cross-hair grid that is situated at the focal plane of the microscope's objective lens. The grid has a diameter of $30 \mathrm{~mm}$. This ensures that the region illuminated by the microscope's light source is covered when an objective lens of $250 \mathrm{~mm}$ focal length is used. Next, the left and right displayed images are centred by superimposing the grid. A coarse alignment is accomplished at low magnification factors $(x 6)$ using $x-y$ translation of the camera coupler and then fine alignment is achieved at higher magnification factors $(x 16, x 25$ and $x 40$ ). Correct alignment of the two monoscopic images is achieved only when they overlay each other in the final display. When alignment is complete, the two couplers are locked at this fixed position.

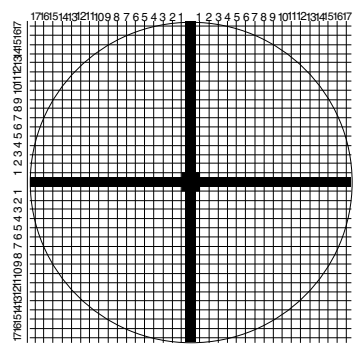

Figure 4: Calibration grid (numbers denote $\mathrm{mm}$ distance from the grid's origin) 
Finally, the passive robot arm is calibrated. This is done by measuring 27 points on the circumference of the 1 -inch sphere with the arm's probe. The $x-y-z$ co-ordinates of the probe location are displayed on the PC screen [5].

\subsection{The Experimental Protocol}

Each operator starts the experiment by trying to identify 3 marked points on the object under the microscope via the autostereoscopic 3D display. He then attempts to bring the arm's probe in the focusing region of the microscope. The probe is not expected to make contact with the designated points on the object. This ensures that the positioning of the probe is driven by visual feedback and not by a motor reflex due to touching. To correctly register a 'hit', the $x-y-z$ position is recorded by pressing a button on the probe's handle. In addition, each time the operator registers a point he then must remove the probe from the microscope's viewing region before carrying on with the next one. This prevents the user from getting to same position via 'memory effect'. This procedure is repeated 5 times for each of the designated points. The whole exercise is then repeated again using the microscope eyepieces only. Finally, without the aid of any magnification device, the exercise is done by means of free viewing of the object. The latter gives the operator more depth cues to work with.
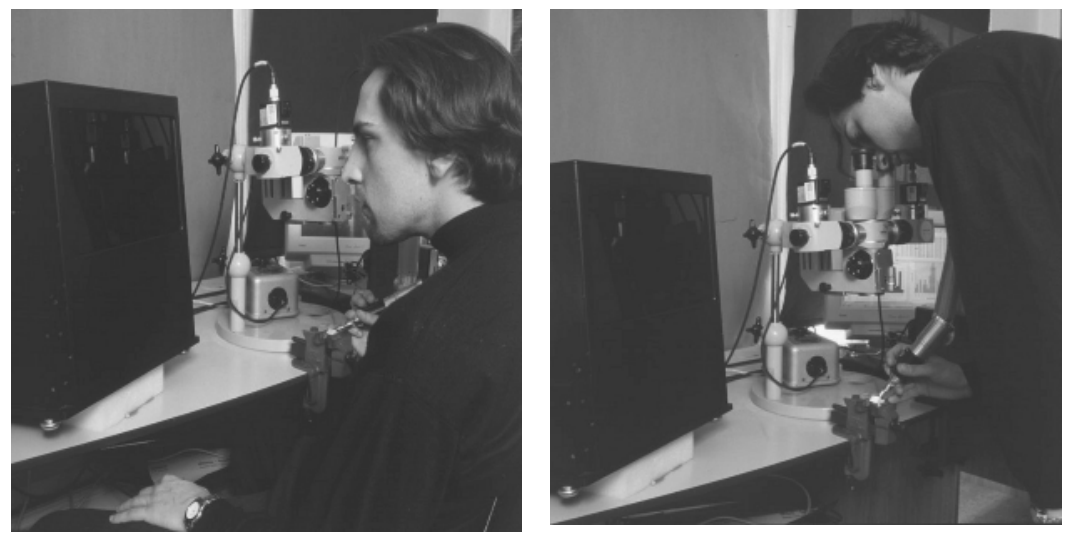

Figure 5: Operator performing the experiment using

(a) the Sharp autostereoscopic 3D display and (b) the microscope eyepieces

The second series of experiments evaluates the time response of each operator when asked to carry out the simple task of passing a thread through the eye of a needle while looking through the eyepieces. This is a representing experiment that takes place at the early stages of microsurgical training. The same experiment is repeated with the examiner looking through the autostereoscopic 3D display and finally, a standard 2D display (i.e. monoscopic display by switching off one of the image channels). 


\section{Results}

\subsection{D Autostereoscopic Display vs Binoculars}

The results showed that there is a correlation between the display and the eyepieces for one of the operators in the $x, y$ and $z$ directions for the second target point on the model. Other graphs for the remaining operators, which are not shown here, exhibit very similar patterns.
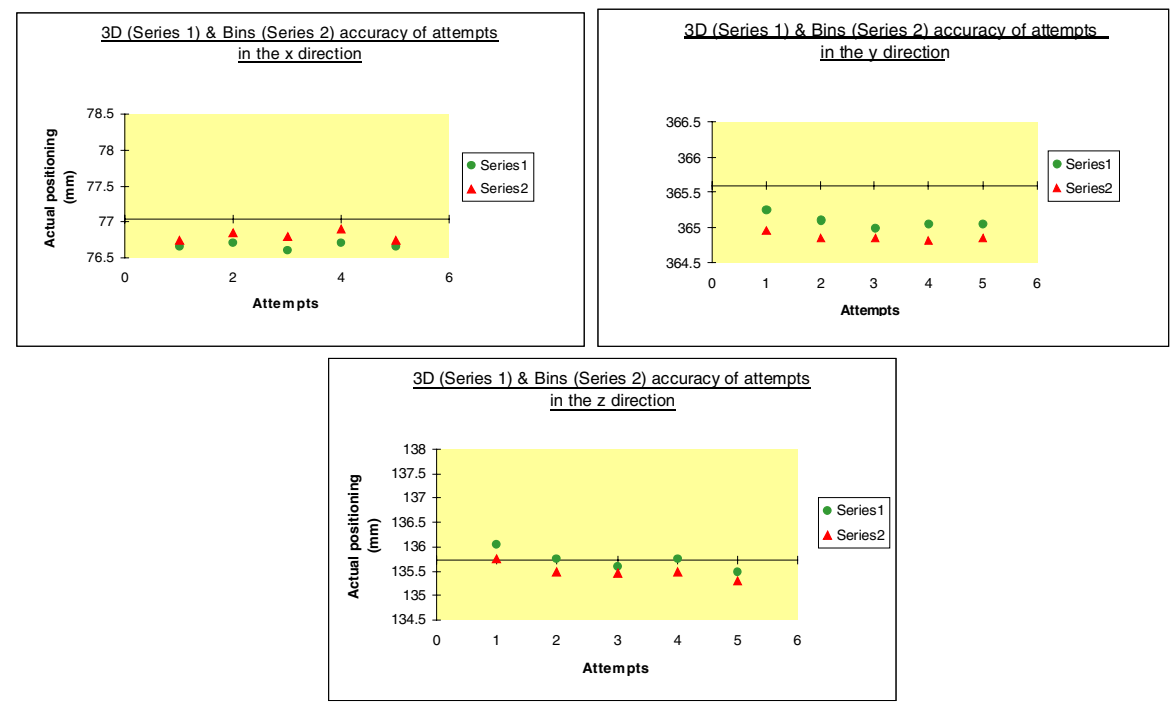

Figure 6

The experiment uses the free viewing value as the gold value from which the other two measuring techniques are compared. In the chart for $\mathrm{x}$ direction above, $77.041 \mathrm{~mm}$ is the gold value while Series 1 represents the deviation using the autostereoscopic display and Series 2 represents the deviation under the binocular eyepieces of the microscope. This 'gold value' is used as a reference value throughout the experiment. Likewise, similar gold values are used for the charts in the $\mathrm{y}$ and $\mathrm{z}$ directions.

The above graphs show little differences between the 3D display and eyepieces measurements for the individual's attempts in 'hitting' the target point. The following figure shows the overall correlation between each pair of results for all operators. This suggests that there is a matching trend in visual perception of the Sharp autostereoscopic 3D display to that achieved by the stereo microscope eyepieces. 


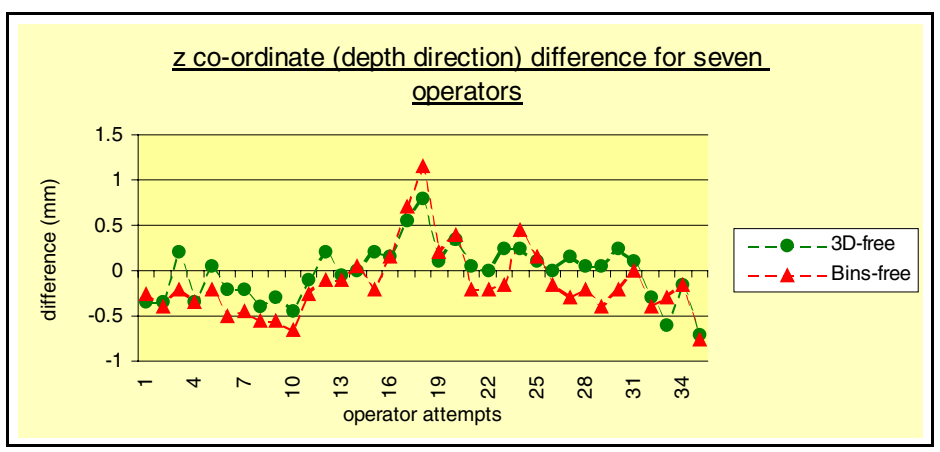

Figure 7: Correlation pattern between the autostereoscopic 3D display and the eyepieces of the microscope.

The overall $x, y$ and $z$ difference for all operators hitting the second marked point is $-0.091 \mathrm{~mm}, 0.064 \mathrm{~mm}$ and $0.121 \mathrm{~mm}$ respectively. This implies good accuracy and precision in readings.

The time-response measurements show clearly the importance of depth information when the operator works in three-dimensional space. Additionally, we notice that the viewers response when using the autostereoscopic 3D display is very similar to when they use the microscope eyepieces.

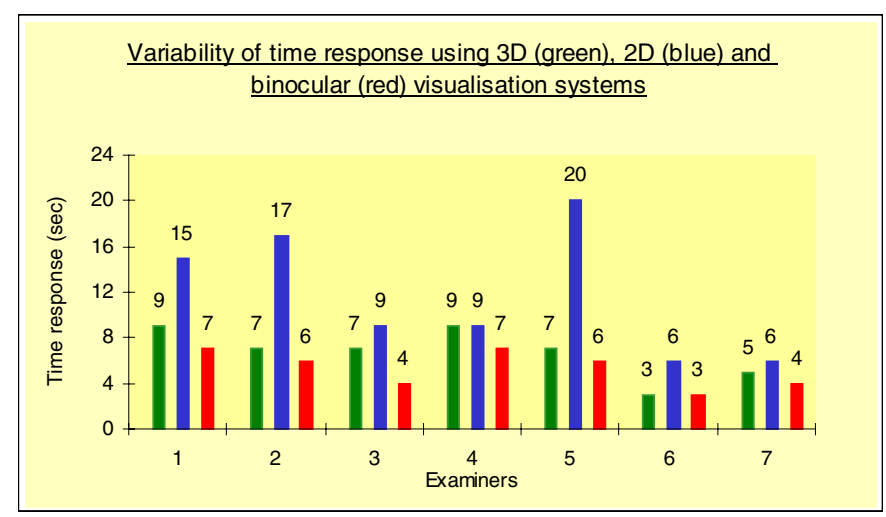

Figure 8: Graphical representation of results from the time study

\subsection{Error Analysis}

In order to validate the accuracy and precision of the Sharp autostereoscopic 3D display we conducted an error analysis. We divided possible sources of error into two categories, statistical and systematic. 


\subsubsection{Statistical Errors}

Statistical errors are due to sample selection. We examined the image perception variance between operators as this occurs when they use the $3 \mathrm{D}$ display or the microscope eyepieces. Figure 9 shows the mean values of the difference in binoculars and $3 \mathrm{D}$ free viewing ( $y$-axis) plotted against the difference in autostereoscopic 3D display and 3D free viewing ( $x$-axis). Each square plotted refers to a different observer.

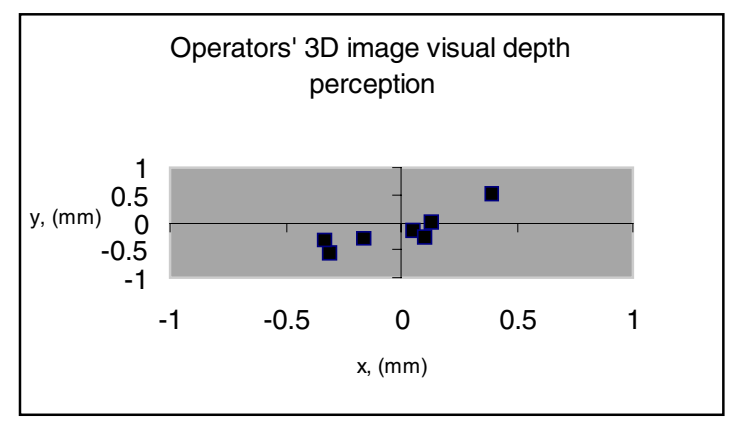

Figure 9

The figure clearly illustrates the way all operators view depth information from both visualisation media. Note that position mean differences are very small with all values lying very close to zero, thus implying only small discrepancies between both the Sharp 3D autostereoscopic display, the binocular stereo microscope with free viewing for each operator. The line along which the points are clustered demonstrates that there is a very small difference indeed between the autostereoscopic 3D display and the binoculars depth perception for each operator in spite of variations in operator bias. The data pattern confirms the fact that different surgeons tend to interpret the image differently. A paired t-test sample confirmed that there was no significant difference between the expected and mean measured values $(t \approx 0.83)$.

\subsubsection{Systematic Error}

Systematic errors are mainly concerned with electronic and electrical deficiencies of the experimental system involved in the measurements. Justifying that the optics involved in the measurements affect both displays in the same fashion we considered errors in the accuracy of the 3D mechanical arm and the depth resolution from the pixel arrangement of the Sharp 3D display.

The active window of our prototype Micro-optic twin-LCD monitor was placed at a distance of $270 \mathrm{~mm}$ from the back LCD panel. Every operator was positioned at $270 \mathrm{~mm}$ from the window plane. At this position the specific prototype offers optimum three-dimensional visual information. The average interpupillary distance of the operators was $60 \mathrm{~mm}$. Mathematical calculations of the Euclidean problem suggest that there is a $0.16 \mathrm{~mm}$ window in the depth axis in which the viewer can see the $3 \mathrm{D}$ image. This error is comparable to the $0.121 \mathrm{~mm}$ error in the operator-response graph of depth perception. 


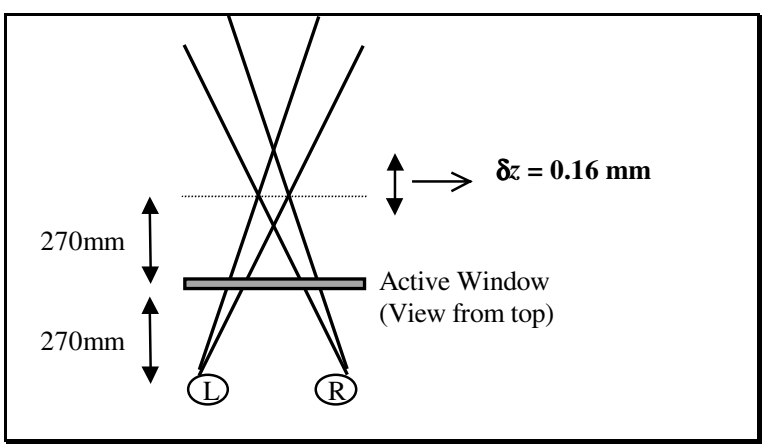

Figure 10: Depth variations in pixel arrangement of display

\section{Discussion}

The results showed that there's a correlation between the viewing properties of the Sharp Micro-optic twin-LCD autostereoscopic display and the eyepieces of the surgical microscope. Great emphasis is given in the perception of depth as this is very important to the acting ENT surgeon while he/she performs a surgical operation. Equally, the results establish the accuracy and precision in viewing three-dimensional objects using the new 'heads up' display.

The initial response of the clinical subjects who tried the experiment was positive to the use of this new viewing device. The device was found to be easily adaptable, accommodating to the eyes and offers natural viewing conditions. Similar results are also noted by other research groups as in laparoscopic [6] and endoscopic [7] surgery. It is envisaged that autostereoscopic 3D displays offer opportunities for lengthy surgeries. The additional benefit of the 'electronic eye' is that the image can also be routed to other display monitors located outside the operating theatre. This would introduce educational benefits for training purposes.

\section{Conclusion/Future Experiment}

At the present time, the use of stereoscopic displays has become a necessity in the practice of microsurgery. In ENT surgery, the use of stereo microscopes is unavoidable but uncomfortable for the surgeon. The system we have configured allows a 'heads up' 3D display where stereoscopic images can be seen in the same way as when viewed through the eyepieces of the microscope.

The experimental data indicate that the accuracy of the system is almost identical to that of the eyepieces. Depth recognition measurements of a microscopic model result to deviations of less than $1 \mathrm{~mm}$, the average surgeon's hand movement precision. 
Also, the time study verified that human observers can accommodate the 3D image from the monitor freely and comfortably. Furthermore, studies for lower magnification levels $(x 10)$ showed similar result with slightly higher discrepancies between the two three-dimensional visualisation media. Finally, we feel that the Sharp autostereoscopic $3 \mathrm{D}$ display can be easily used in the implementation of augmented reality computerassisted surgery.

Our experiment was laboratory-based and tried by seven clinical users. However, we noticed a few variations in the perception of the 3D image between different observers. This implies the possible intervention of psychological factors. Previous studies have shown direct association between surface curvature, and image spatial separation [8]. We will examine how this theory has interfered with our prototype monitor. We are in the process of running clinical trials using the 3D display.

The results of the experiment encourage the use of the Sharp Micro-optic twinLCD monitor for stereo viewing of microsurgical procedures. The system is still only a prototype and work in theatre ergonomics will be needed for the optimum design of the 3D microscope-display system.

\section{Acknowledgements}

Our group would like to thank the Defeating Deafness charity organisation for their financial support of the project. We would also like to thank Sharp Europe Laboratories Ltd. for their constant support, help and assistance with the 3D viewing prototype.

\section{References}

[1] P.J. Bos, Liquid-crystal shutter systems for time multi-plexed stereoscopic displays, D.F. McAllister (Ed.), Stereo Computer Graphics and Other true 3D Technologies, Princetown University Press, Princetown, pp. 90-118, (1993)

[2] Graham J. Woodgate et al, Autostereoscopic 3D display systems with observer tracking, Signal Processing: Image Communication 14, pp. 131-145, (1998)

[3]D. Ezra et al, New autostereoscopic display system, Proc. SPIE 2409, pp. 31-40 February 1995 ,

[4]FaroArm Silver 8, Faro Technologies Inc., Florida, USA

[5] Caliper 3D for Windows manual, Faro Technologies Inc., Florida, USA, (1997)

[6]N.A. Dodgson et al, Autostereoscopic 3D Display in Laparoscopic Surgery, Proc of CAR'95, H U Lemke et al (Ed.), Springer-Verlag Berlin, pp. 1139-1144, (1995)

[7]J. Woodham, Amin Javer, "Heads-Up-FESS"- working from the TV monitor, FESS Special Feature, ENT News, vol. 7, no. 5, pp. 19-20, November/December 1998

[8] W. Curran, A. Johnston, Three-dimensional Curvature Contrast-Geometric or Brightness Illusion?, Vision Res., vol. 36, no. 22, pp. 3641-3653, (1996) 